\title{
Philosophiques
}

\section{Le statut du serment et de la promesse dans la déclaration des droits de 1789}

\section{Christine Fauré}

Volume 19, numéro 1, printemps 1992

URI : https://id.erudit.org/iderudit/027173ar

DOI : https://doi.org/10.7202/027173ar

Aller au sommaire du numéro

Éditeur(s)

Société de philosophie du Québec

ISSN

0316-2923 (imprimé)

1492-1391 (numérique)

Découvrir la revue

Citer cet article

Fauré, C. (1992). Le statut du serment et de la promesse dans la déclaration des droits de 1789. Philosophiques, 19(1), 75-85. https://doi.org/10.7202/027173ar
Résumé de l'article

Le sens et la fonction de la Déclaration des droits de l'homme et du citoyen furent l'objet de longues et nombreuses controverses. Parmi les différentes approches du texte, juridiques ou sociologiques, nous retiendrons la méthode conçue à propos de Ténoncé performatif par le philosophe anglais J. L. Austin, car elle nous permet de découvrir sur quelles procédures conventionnelles s'appuie la bonne réception de la Déclaration, à quelles règles de conduite existantes, les constituants ont-ils fait appel. Ces conventions, en l'occurrence l'acte de serment et de promesse, apparaissent dans le texte à l'état d'indices, cette dimension étant masquée par l'intensité de la rupture que la Déclaration instaure au plan politique. Ce décodage des intentions des constituants nécessite le recours à une analyse des contextes: contexte socio-historique immédiat et environnement conceptuel. 


\section{LE STATUT DU SERMENT ET DE LA PROMESSE DANS LA DÉCLARATION DES DROITS DE 1789 \\ par Christine Fauré}

Résumé. Le sens et la fonction de la Déclaration des droits de l'homme et du citoyen furent l'objet de longues et nombreuses controverses. Parmi les différentes approches du texte, juridiques ou sociologiques, nous retiendrons la méthode conçue à propos de l'énoncé performatif par le philosophe anglais J. L. Austin, car elle nous permet de découvrir sur quelles procédures conventionnelles s'appuie la bonne réception de la Déclaration, à quelles règles de conduite existantes, les constituants ontils fait appel. Ces conventions, en l'occurrence l'acte de serment et de promesse, apparaissent dans le texte à l'état d'indices, cette dimension étant masquée par l'intensité de la rupture que la Déclaration instaure au plan politique. Ce décodage des intentions des constituants nécessite le recours à une analyse des contextes: contexte socio-historique immédiat et environnement conceptuel.

ABSTRACT. The meaning and the function of the Declaration of the rights of Men and Citizen has been discussed for a long time. We prefer, among different lectures (juridical and sociological) of the text, the method contrived for an explanation of performative utterances, by the english philosopher J. L. Austin: it can enable us to discover on what convention a good reception of the Declaration is based, what rules of conducts the " constituants " have choosed. These conventions - oath and promise - appear in the text as mere indications. The intensity of the political rupture hide them. To decode the " constituant's " intentions, a contextual analysis is needed, that is both immediate socio-historical context and conceptual context. 
La rédaction de la Déclaration des Droits de 1789 a donné lieu, en juillet et août 1789, à l'Assemblée Constituante, à deux sortes de débats: la Déclaration des Droits faisait-elle partie de la constitution, en était-elle distincte, devait-on la placer avant ou après la Constitution? Le modèle américain ${ }^{2}$ n'apportait pas de réponse suffisamment convaincante sur le lien entre Déclaration et Constitution à ces constituants, souvent légistes, épris $d^{\prime}$ une rigueur formaliste. Le second volet des débats portait sur le contenu des articles qui furent rédigés, amendés et votés en l'espace de six jours, l'un après l'autre, sans qu'il y ait eu récapitulatif. La Déclaration des Droits de 1789 est un texte inachevé é $^{3}$, un texte dont la rédaction fut interrompue le 27 août 1789 et jamais reprise.

Ces quelques remarques nous introduisent à la question du sens et de l'interprétation de la Déclaration des Droits de 1789. Est-elle encore un énoncé de principe, est-elle pourvue d'une efficacité sociale, a-t-elle une valeur juridique? En France, contrairement aux États-Unis où la constitution fédérale a été rédigée une fois pour toutes, le grand nombre des déclarations, presque à chaque nouvelle constitution (en 1793; 1795; 1848; 1946) n'a-t-il pas relativisé le pouvoir de constitution qu'on lui prêtait en 1789? Ces questions furent l'objet de controverses qui dépassèrent la période révolutionnaire. Elles culminèrent dans des polémiques menées par ces très grands théoriciens du droit que furent Raymond Carré de Malberg ${ }^{4}$, Léon Duguit ${ }^{5}$, Adhémar Esmein ${ }^{6}$ et Hauriou ${ }^{7}$. Carré de Malberg écrit dans sa remarquable "Théorie générale du droit»:

La Déclaration n'est pas, à proprement parler, une déclaration de droits, mais seulement une déclaration de principe: elle ne formule pas de règles juridiques qui soient susceptibles d'être appliquées pratiquement par un juge; elle ne met pas les citoyens en état de faire valoir devant les tribunaux telle ou telle faculté individuelle nettement délimitée ${ }^{8}$.

Il fallut attendre la Constitution d'octobre de 1946 et surtout, la mise en place d'un contrôle constitutionnel des lois, pour que la prééminence de la Déclaration de 1789 soit affirmée. Les actes de barbarie perpétrés pendant la seconde guerre mondiale avaient rendu nécessaire ce retour aux énoncés du droit naturel. La question du sens et de l'interprétation de la Déclaration fut également l'objet d'un autre genre d'argumentation, fort répandu puisqu'il résuma longtemps l'opinion du monde anglophone sur le texte de 1789. L'énoncé de la Déclaration est absurde en regard de la situation sociale à laquelle il se 
réfère: "All men are born free? All men remain free? No; not a single man, not a single man that ever was, is or will be. All men on the contrary are born in subjection and the most absolute subjection. The subjection of a helpless child to the parents ", écrit Jeremy Bentham ${ }^{9}$. L'égalité naturelle n'existe pas. La hiérarchie des états est un fait patent de nos sociétés. La déclaration des droits est une dénégation de la réalité.

On peut accepter avec Bentham l'idée que la Déclaration des Droits ne constate rien de réel, mais pour en venir à de toutes autres conclusions. La Déclaration des Droits n'est pas un énoncé qui délivre une information vérifiable. Elle ne décrit rien. Elle appartient à un autre registre du langage, à la catégorie du «speech act " élaborée par J. L. Austin ${ }^{10}$.

Alors que la théorie du " dire action », pensée en Angleterre dans les années 1960, connut de multiples applications en littérature, mais aussi en sciences politiques ${ }^{11}$, on songea très récemment à l'appliquer en France ${ }^{12}$ pour renouveler l'interprétation de la Déclaration des Droits, à la lumière notamment d'une analyse contextuelle de sa rédaction. Cette approche, conçue par Austin, avait pour caractéristique essentielle de traiter des énoncés relevant du langage ordinaire, bien que, dans un premier temps, il y eut quelque intérêt pour les procédures conventionnelles et juridiques ${ }^{13}$. Elle tentait de déterminer, avec autant de précision possible, l'intention des acteurs par rapport à leur propre énoncé. L'analyse de l'intention fait intervenir la connaissance de la norme en vigueur, tout en pariant sur l'avenir et en projetant un résultat. Une symétrie est établie entre la signification de l'énoncé et l'acte du sujet. Traiter la Déclaration des Droits comme un « speech act » revient à mettre en évidence les conditions nécessaires à son fonctionnement " heureux " pour reprendre la formule d'Austin. Parmi ces conditions, l'existence d'une procédure reconnue, le choix des personnes autorisées, et des circonstances adéquates. La difficulté majeure concernant ce traitement de la Déclaration des Droits provient du fait que son caractère inaugural masque le conventionnalisme de la démarche. C'est la première fois qu'en France, on rédige une Déclaration de Droits. Elle représente une rupture avec les formes précédentes de gouvernement. Mais, pour rendre efficace leur volonté de changement, les constituants ont choisi dans le stock des conventions existantes, une procédure reconnue par leurs contemporains, et qui en garantit la compréhension et le consentement de tous. 
La formule "en présence et sous les auspices" de l'être suprême ${ }^{14}$ fut souvent considérée comme la marque d'un habillage solennel. Pourtant, par ces mots, l'Assemblée Nationale prend à témoin la Divinité de sa déclaration. Cette prise à témoin est similaire à l'acte de prêter serment: le respect de l'énoncé devient fonction de la croyance des députés en l'être suprême, comme dans le serment traditionnel inviolable à cause de l'attestation divine. Cependant, souligner cette similitude ne suffit pas. Il faut en démontrer la pertinence à travers une étude des contextes de la Déclaration, contexte socio-historique immédiat, contexte d'ordre conceptuel pour ensuite revenir à la lettre même du texte.

\section{Le contexte promissif de la Déclaration des Droits de $\mathbf{1 7 8 9}$}

La Déclaration des Droits de l'homme qui fut la première production d'importance de l'Assemblée Constituante, fait suite à des manifestations qu'il convient de mettre en perspectives, du célèbre texte. Parmi ces manifestations, le Serment du Jeu de Paume dont on connaît la fameuse interprétation de David, déjà auteur d'un Serment des Horaces (1785).

L'histoire de ce Serment s'inscrit dans les multiples résistances qu'opposa Louis XVI à la reconnaissance de la réunion des trois ordres qui venait de prendre le nom d'Assemblée Nationale (le 17 juin 1789). Le 20 juin 1789 les portes de la salle de réunion des menus-plaisirs étaient closes sous prétexte de préparatifs à la séance royale des États où le souverain devait reprendre l'initiative face aux prétentions des députés; Les députés se réunirent donc dans une salle voisine, la salle du Jeu de Paume et y prêtèrent serment: "l'Assemblée nationale, considérant qu'appelée à fixer la Constitution du Royaume, arrête que tous les membres de cette Assemblée prêtent à l'instant serment solennel de ne jamais se séparer et de se rassembler partout jusqu'à ce que la Constitution du royaume soit établie et affermie sur des fondements solides $»^{15}$. Cet acte de serment fut occasionnel. Il affirme la mission des députés en quelque lieu que ce soit, il renforce les liens de solidarité entre les membres de l'Assemblée Nationale et consacre l'autonomie de leur pouvoir par rapport à la volonté et au regard $\mathrm{du}$ roi. Le Serment du Jeu de Paume traduisait un surcroît d'obligation pour ces représentants des États-Généraux qui avaient déjà dû prêter serment au roi, lors de leur prise de fonction: "Je promets et je jure devant Dieu, sur les saints Évangiles, de dire tout ce que je penserai en ma conscience 
être de l'honneur de Dieu, le bien de son Église, le service du Roi et le repos de l'État $»^{16}$. À travers les débats de l'Assemblée Constituante, est clairement exprimée la volonté de caractériser le Serment, acte fort répandu dans tous les secteurs de la vie sociale.

On opposait le Serment ${ }^{17}$ qui lie le soldat à sa hiérarchie, au point de le faire agir contre les devoirs de l'homme, à une autre forme d'adhésion, fondée sur la reconnaissance de l'autre comme égale et non sur la soumission. En l'espèce, le Serment se trouvait réduit à une obligation sans choix préalable, compte tenu des conditions de l'enrôlement, à un résidu du despotisme royal. On pourrait multiplier les exemples concernant la pratique du Serment. Ils découvrent, par approximations successives, des formes singulières d'adhésion et de reconnaissance. En outre, ils vulgarisent l'hypothèse savante, selon laquelle l'existence de personnes libres, donnant accord dans des circonstances convenues, déterminerait une nouvelle règle de vie comportant des droits et des devoirs.

\section{Les formes de la parole donnée et le droit naturel}

Prenons les définitions de l'Encyclopédie comme axe de comparaison. Que nous disent-elles? L'Encyclopédie (1751-1780), comme à son habitude, recense les divers usages et propose des définitions distinctes du Serment et de la Promesse. «Le Serment, $c^{\prime}$ est à l'homme qu'on s'engage... On prend seulement Dieu à témoin de ce à quoi on $s^{\prime}$ engage et l'on se soumet aux effets de la vengeance. Le serment est l'attestation religieuse de la vérité ${ }^{18}$. Les références à $l^{\prime}$ Antiquité grecque, latine, perse, hébraïque, sont nombreuses, mais il est également dit que le Serment est l'instrument qu'a privilégié la hiérarchie féodale pour faire respecter son ordre. "Ignoré des premiers hommes", son invention fut rendue nécessaire par la déchéance de la condition humaine marquée par le mensonge et la fraude. L'histoire du serment, selon l'Encyclopédie, suit de près le dogme du péché originel. La promesse à l'inverse, est un engagement laïque qui peut prendre deux formes: forme verbale solennisée par des paroles rituelles ou écrites entre deux personnes privées, sous-seing privé ou devant notaire. On les appelle alors ubligation ou contrat. La promesse engage l'avenir. Les conditions de son respect sont à la mesure de l'attente qu'elle soulève chez l'autre. L'obligation est relative aux sentiments du partenaire. C'est donc à un corpus de définitions relativement éclatées que nous avons affaire dans l’Encyclopédie. Ce corpus 
ignore toutes interférences subvenues entre les notions qui pourtant, avaient largement précédé sa rédaction, et qui avaient marqué le travail des théoriciens du droit naturel. L'originalité de Grotius et de Pufendorf dans ce domaine, est d'avoir tenté de formuler un modèle unique. Le droit de la guerre et de la paix ${ }^{19}$ et le droit de la nature et des gens ${ }^{20}$ furent des ouvrages majeurs dans l'élaboration du droit naturel, vulgarisés au XVIIIe siècle en France par les traductions de Jean Barbeyrac. Le commentaire formaliste des usages sociaux, traditionnel pour les jurisconsultes, coïncide avec la recherche des éléments fondateurs d'une société plus juste. De quelle manière cette coïncidence fut-elle aménagée? Les deux auteurs distinguent, comme il se doit, le serment de la promesse. Tout en leur reconnaissant leur spécificité, un modèle unique se met peu à peu en place. Grotius consacre un chapitre au Serment. C'est une notion lourde que l'auteur aborde par le statut social du parjure. Peut-on rompre un serment? à quelles conditions? Y a-t-il des serments qui n'ont pas force d'obligation? En fait, le serment est considéré par l'auteur comme un acte de langage qui exige pour son accomplissement, un certain nombre de conditions: "Afin qu'un serment soit valable, il faut que l'on ait pu s'engager en conscience à ce que l'on a juré de faire ou de ne pas faire $»^{21}$. Le caractère automatique de l'obligation d'un serment, lié à la simple énonciation du nom de Dieu, est mis à distance. Prononcer un serment devient un acte profondément rationnel. "Il faut d'abord supposer ici, comme dans les promesses et les contrats, que celui qui jure, ait l'usage de la raison et qu'il agisse avec délibération ${ }^{22}$. L'important, $c^{\prime}$ est de remplir les conditions de l'acte pour qu'il s'effectue correctement. La réalité de l'engagement, son caractère vraisemblable lui sont donnés par la clarté de l'énoncé et restent indépendants de l'acte d'invocation. "Il n'est pas nécessaire de parler des serments qui regardent quelque chose d'impossible. Car il est clair que personne n'est tenu à ce qui est absolument impossible ${ }^{23}$.

Le serment est une forme de parole donnée qui prend à témoin la divinité quelle qu'elle soit, vraie ou fausse. En ce sens, Grotius s'emploie à détacher l'acte de langage de son contexte religieux. "Les serments même faits par de faux dieux, ne laissent pas d'obliger ceux qui les font ${ }^{24}$. Pufendorf accentue le mouvement de laïcisation du serment. Par rapport à la position de Grotius, une étape est franchie. La correction de l'énoncé prime définitivement: "Nul acte où il y a quelque 
vice qui le rend incapable de produire aucune obligation, ne devient obligatoire par l'interposition du serment... Ainsi l'on a beau jurer de ne pas payer une dette, on n'est pas pour cela quitte avec le créancier $"{ }^{25}$, conclut-il avec humour. Dans les deux cas de serment et de promesse les conditions d'annulation sont identiques. La promesse comme le serment, implique un usage de la raison. "L'insensé", "le furieux", mais aussi $l^{\prime} e n f a n{ }^{26}$, sont écartés de la possibilité de prononcer des promesses. Certains états provisoires que connaissent des êtres de raison, peuvent contribuer à rendre nulle une convention entre deux personnes: l'ivresse, mais aussi la crainte, pour Pufendorf se séparant en cela de l'avis de Grotius, qui croyait qu'on était obligé de tenir les promesses extorquées par la peur. La qualité morale des contractants est décisive dans le respect de la parole donnée. Le personnage du corsaire, l'ennemi public par excellence pour Grotius et Pufendorf, justifie tous les manquements à cette parole. L'acte de langage ne se définit pas seulement par l'état dans lequel se trouve le locuteur, par la clarté de ses intentions et de sa volonté, mais aussi par «la production d'un signe extérieur à cette volonté", pour reprendre une formule de Pufendorf. L'extériorité de l'acte de langage revient à utiliser une codification déjà là, sur laquelle il ne doit pas y avoir de doute et d'erreur. La démarche des jurisconsultes cependant, n'est pas seulement descriptive, mais aussi spéculative. Le répertoire des actes classés selon leur nature et les circonstances dans lesquelles ils s'accomplissent, n'a de valeur que par rapport à un postulat de départ, établir les termes d'une obligation loyale, c'est préciser la possibilité de l'existence d'une société compatible avec l'égalité naturelle des hommes.

Grotius écrit dans la préface de son ouvrage: «il est de droit naturel que chacun tienne religieusement sa parole (car il était nécessaire qu'il y eut parmi les hommes, quelque matière de s'engager les uns aux autres) ${ }^{27}$. Pour Pufendorf, encore plus nettement, il y a fusion entre l'obligation de se regarder les uns et les autres, comme naturellement égaux, et l'obligation de transparence qui concerne l'usage de la parole. "La vérité consiste donc à faire en sorte que les signes extérieurs dont on se sert, et surtout les paroles, représentent fidèlement nos pensées à ceux qui ont droit de les connaître et auxquels nous sommes tenus de les découvrir en vertu d'une obligation parfaite ou imparfaite ${ }^{28}$. Cette capacité d'obligation est un élément de distinction entre la société humaine et la société 
animale. "Il ne peut y avoir naturellement, aucun droit, ni aucune obligation commune aux hommes et aux bêtes " ${ }^{29}$. La possibilité d'un engagement mutuel est fondatrice de société dans le droit naturel. Peut-on risquer l'hypothèse que le concept de droit naturel soit assimilable à cet acte de langage, correctement effectué: tenir sa promesse. Cet acte de langage assure la stabilité d'une société quelle qu'elle soit et symbolise la bonne entente de ses membres.

\section{L'autre comme soi dans la Déclaration des Droits}

L'acte de promettre, nous l'avons vu, joint d'un statut paradigmatique dans la constitution de la doctrine du droit naturel, et les droits naturels dont il est fait mention dans le préambule de la Déclaration et à ses articles I, II, IV et VI témoignent encore de cet acte de langage qui s'est développé et hissé au niveau d'une philosophie. Il est cependant dans le texte de la Déclaration, la marque d'un conventionnalisme, souvent passé inaperçu et propre à étayer notre thèse. Dans le préambule de la Déclaration, il est fait référence à une déclaration qui est en train de se faire: "Les représentants du peuple français [...] ont résolu d'exposer dans une Déclaration solennelle... en conséquence, l'Assemblée Nationale reconnaît et déclare [...]"

Ce caractère d'auto-référentialité reprend le mouvement réflexif qu'implique la reconnaissance de l'autre comme soi dans la promesse. Il rejoint également un trait de l'énoncé performatif maintes fois souligné par les linguistes ${ }^{30}$ : «se référer à une réalité qu'il constitue lui-même ". Autant dire que nous avons là trois cas d'auto-référentialité que nous pouvons ramener à un phénomène unique. L'auto-réflexivité est le propre de toute procédure conventionnelle orale et écrite. La formule consacrée des actes notariés "par la présente, je m'engage... » ne signifie pas autre chose. Pour être compris, pour enlever l'adhésion des destinataires, les constituants ont utilisé des conventions qui appartenaient au secteur de la vie privée. Avec la Déclaration des Droits, il y a transfert à la vie publique de ces formes, serments ou promesses, qui arbitrent les relations entre les hommes et dans lesquelles la confiance de tous est directement engagée.

À partir de cette analyse des formes de la parole donnée, on peut renouveler l'interprétation de l'universalité de la Déclaration des Droits, puisque toute société pour survivre doit s'assurer une stabilité dans les échanges entre individus. Ces remarques 
ont néanmoins pour objectif de couper court à une formulation métaphysique du droit naturel qui relègue les Déclarations des droits dans le champ d'une éthique dépourvue de tout efficacité. Elles ont également pour but d'affaiblir une opposition ressassée entre utilitaristes et partisans du droit naturel, que les malentendus culturels entre anglo-saxons et continentaux ont hypertrophiée. David Hume ${ }^{31}$ par exemple, en liant l'obligation de tenir sa promesse à l'utilité publique, s'en est pris avec vigueur aux partisans du droit naturel: les promesses sont honorées dans la mesure où elles servent les intérêts de la société, sa sécurité et sa protection. C'est une affaire de convention: "toute promesse serait incompréhensible hors du contexte institutionnel qui l'a établie ». La caractéristique de la démarche de Hume réside dans la prise en compte des mécanismes psycho-moraux qui fonde l'obligation. Les hommes respectent leur promesse, non pas en fonction d'une hypothétique réciprocité, mais pour des intérêts personnels égoïstes qui relèvent de l'exigence de servir. L'exposé présenté ici peut constituer un embryon de réponse aux allégations provocatrices de Hume qui firent recette Outre-Manche au fil des siècles. L'hypothétique réciprocité avancée par les théoriciens du droit naturel est en fait fondée sur la reconnaissance des Conventions préexistantes à travers un acte de langage spécifique. Cet acte de langage intervient au coeur d'une relation intersubjective, régulée par les exigences d'une compréhension mutuelle. En conséquence, les droits naturels que mentionne la Déclaration des Droits, ne puisent pas leur légitimité à l'extérieur de l'édifice social, par un recours à un principe transcendant. La combinaison $d^{\prime}$ un modèle serment-promesse joue un rôle constitutif dans l'élaboration du droit naturel et dans les formes d'obligation instaurées en son nom.

Directeur de Recherches au C.N.R.S. en sociologie Centre de sociologie de l'éthique-E.H.E.S.S., Paris

\section{NOTES}

1. Archives parlementaires, Paris, Imprimerie Nationale, 1875, Tome VIII, 1ère série - A. de Baecque, W. Schmale, M. Vovelle, L'an 1 des droits de l'homme, Paris CNRS, 1988.

2. Les textes américains et notamment la Déclaration des Droits de Virginie avaient fait l'objet d'une publication en langue française 
dans les affaires de l'Angleterre et de l'Amérique de 1776-1778. Anvers, Paris. Ils avaient été réunis dans une édition spéciale, traduite par L.A. de la Rochefoucault d'Anville: "Constitution des Treize États-Unis de l'Amérique ", Philadelphie, Paris, Pierres, 1783. Il semble pourtant que les constituants ne connaissaient pas le débat en cours qui partageait l'opinion américaine, sur la nécessité d'un bill of rights dans la Constitution fédérale (1787-1791).

3. Ch. Fauré, Les déclarations des droits de l'homme de 1789, Paris, Payot, 1988; édition de poche, 1992, p. 16.

4. R. Carré de Malberg, Contribution à la théorie générale de l'État, Paris, recueil Sirey, 1920; CNRS 1962/1985.

5. L. Duguit, Traité de droit constitutionnel, Paris, Boccard, 1938.

6. A. Esmein, Éléments de droit constitutionnel français et comparé, Paris, Larose, 1899.

7. M. Hauriou, Précis de droit administratif et de droit public, Paris, recueil Larose 1892, Sirey, 1933.

8. R. Carré de Malberg, op. cit. Tome II, p. 581.

9. J. Bentham, Anarchical fallacies in "Nonsense upon stilts", ed. by J. Waldron, London, Methuen, 1987, p. 49.

10. J. L. Austin, Quand dire, c'est faire, Paris, Seuil, 1970.

11. J. Tully, Meaning and context. Quentin Skinner and his critic Cambridge, Polity press, 1988.

12. G. Soulier, "Le sens de la déclaration des droits de l'homme", dans Le Français aujourd'hui, association française des Enseignants de français, juin 1988; Ch. Fauré, op. cit.; A. Trognon, «La déclaration en tant qu'acte de discours " in Les valeurs de la Révolution devant la science actuelle, sous la direction de F. Borella, Nancy, Presses Universitaires de Nancy, 1990.

13. Ch. Fauré “Ce que déclarer des droits veut dire, l'exemple de la Déclaration des Droits de l'homme et du citoyen de 1789 ", Cahiers internationaux de sociologie, vol. XCI, 1991, p. 267.

14. Ch. Fauré, «La déclaration des droits de 1789: le sacre et l'individuel dans le succès de l'acte ", dans La Déclaration des droits de l'homme et du citoyen de 1789, ses origines, sa pérennité, Paris, La Documentation Française, 1990.

15. Archives parlementaires, op. cit., p. $\mathbf{1 3 8}$.

16. Ibidem, op. cit., p. 203.

17. Discours de Duval d'Epremesnil, député de la noblesse de la prévôté de Paris, dans Archives parlementaires, op. cit., p. 377.

18. L'Encyclopédie ou Dictionnaire raisonné des sciences, des arts et des métiers, volume 15, Stuttgart, Bad Cannstatt, 1967, pp. 99-100. 
19. Hugues Grotius, Le Droit de la Guerre et de la Paix, nouvelle traduction par Jean Barbeyrac, [Amsterdam, Pierre de Cour 1724], Bibliothèque de Philosophie politique et juridique, coll. " textes et documents ", Université de Caen, 1984.

20. Samuel Pufendorf, Le droit de la nature et des gens ou système général des principes les plus importants de la morale, de la jurisprudence et de la politique, Amsterdam, Gérard Kuyper, 1706.

21. Hugues Grotius, op. cit., livre II, ch. XIII, $\S$ VI.

22. Hugues Grotius, op. cit., livre II, ch. XIII, § II.

23. Hugues Grotius, op. cit., livre II, ch. XIII, $\S$ VIII.

24. Hugues Grotius, op. cit., livre II, ch. XIII, $\S$ XII.

25. Samuel Pufendorf, op. cit., livre IV, ch. II, § VI.

26. Hugues Grotius, op. cit., livre II, ch. XI, $\S$ V.

27. Hugues Grotius, op. cit., discours préliminaire, $\S \times V$.

28. Samuel Pufendorf, op. cit., livre IV, ch. I, § VIII.

29. Samuel Pufendorf, op. cit., livre IV, ch. III, $\S$ V.

30. Émile Benveniste, Problème de linguistique générale, Paris, Gallimard. 1966-1974, p. 274, Tome 1.

31. David Hume, Traité de la nature humaine, Paris, Aubier 1989, p. 635. 